\title{
Pay for Performance: Was ist machbar? Sicht der Bundesärztekammer
}

\section{R. Klakow-Franck}

Qualitätsmanagement

Schlüsselwörter

Pay for Performance

$\checkmark$ Qualitätsverbesserung

qualitätsabhängige

Vergütung

Anreize

Keywords

pay for performance

quality improvement

quality

based-reimbursement

Dincentives

Institut

Bundesärztekammer, Berlin

Bibliografie

Dol 10.1055/s-0029-1242677

Dtsch Med Wochenschr 2009;

134: S322 - (c) Georg Thieme

Verlag KG Stuttgart · New York ISSN 0012-0472

Korrespondenz

Dr. med. Regina Klakow-

Franck, $M$. A.

Bundesärztekammer

Postfach 120864

10598 Berlin

Tel. 030/400456-430

eMail regina.klakow-franck@

baek.de
P4P - Versorgungssteuerungsmodell Nr. 1? In der gegenwärtigen gesundheitspolitischen Diskussion in Deutschland ist „Pay for Performance“ (P4P) in aller Munde und wird zum Versorgungssteuerungsmodell Nummer 1 hochstilisiert. Dabei dürften die Vorstellungen, was unter „Performance“, aber auch, was unter „Pay“ zu verstehen ist, weit auseinander liegen. Während sich aus der Perspektive der Krankenhäuser und Ärzteschaft mit P4P Hoffnungen auf zusätzliche Belohnung für besonders gute Qualität verbinden, zielen die Krankenkassen auf Marktbereinigung und Ausschöpfung von Effizienz- und Wirtschaftlichkeitsreserven durch kostenneutrale Umverteilung der Vergütung. Aus Patientenperspektive liegt der Fokus auf Gewährleistung von Qualitätsmindeststandards und Patientensicherheit in einem stärker marktorientierten Gesundheitswesen.

\section{Pay for Outcome?}

Eine weitere Besonderheit der deutschen P4P-Debatte besteht darin, dass das Spektrum von belohnungswürdiger „Performance“ sehr stark auf medizinische Ergebnisqualität eingeengt wird, während ausländische P4P-Programme Anreize auch für Prozess- und Strukturqualitätsverbesserungen, Erhöhung der Patientenzufriedenheit oder auch für IT-Investitionen ausloben. Medizinische Ergebnisqualität kann im Hinblick auf Qualitätssicherung und Qualitätsdarstellung (einschließlich öffentlicher Qualitätsvergleiche) wohl als die anspruchvollste der 3 Qualitätsdimensionen gelten. Eine der zentralen Voraussetzungen für eine patientenzentrierte Outcome-Qualitätssicherung, die sektorenübergreifende Qualitätssicherung, steckt jedoch noch in den Kinderschuhen.

\section{Kein Anreizsystem ohne eigene Fehlanrei- ze}

P4P kann zur Erreichung all der oben genannten Erwartungen sowie allgemein zur Qualitätsentwicklung im deutschen Gesundheitswesen beitragen - jedoch nicht im Sinne eines monokausalen Lösungsansatzes, sondern in Kombination und Wechsel mit anderen Anreizsystemen.

P4P stellt ein Instrument unter vielen dar, um ökonomischen Fehlanreizen pauschalierter Vergütungssysteme entgegenzuwirken, wie sie im letzten Jahrzehnt im GKV-System etabliert wurden, sei es in Gestalt des DRG-FallpauschalenEntgeltsystems oder des stark pauschalierten vertragsärztlichen EBM. Es gibt jedoch kein Anreizsystem, das seinerseits nicht das Risiko von Fehlanreizen in sich trägt. Die Risiken von P4P sind bekannt: Patientenselektion, Förderung von
Prozesskonformität statt tatsächlicher Qualitätsverbesserung, Manipulation von Daten und Codierung etc. [1]. Der Aufwand (Kosten und Bürokratie) für Implementierung, Kontrolle und $\mathrm{Ge}$ gensteuerung von Fehlentwicklungen von $\mathrm{P} 4 \mathrm{P}$ ist beträchtlich und an einen lückenlosen Datentransfer zwischen Leistungserbringer und Krankenkasse gebunden.

\section{Notwendige Rahmenbedingungen und} sinnvolle Einsatzmöglichkeiten für P4P

Geld gilt allgemein zwar als erfolgreichstes Erziehungsinstrument bei Erwachsenen - P4P aber bleibt wirkungslos, wenn es nicht eingebettet ist in eine qualitätsorientierte Unternehmenskultur. Die auf Ebene der Versorgungseinrichtungen gemachte Beobachtung, dass zu den „Top Performern“ regelmäßig jene Krankenhäuser zählen, die sich durch eine einrichtungsinterne Qualitätskultur über alle Hierarchie-Ebenen hinweg sowie ein funktionierendes Qualitätsmanagement auszeichnen [2], sollte eins zu eins bei der Gestaltung der Rahmenbedingungen medizinischer Versorgung beherzigt werden:

Monetäre Anreizsysteme wie P4P können die notwendige Eigenmotivation und Verantwortungsbereitschaft - neudeutsch „Accountability“ [3] - der Ärztinnen und Ärzte und der nichtärztlichen Gesundheitsberufe nicht ersetzen, sondern sind, wenn eine Wirkung im Sinne einer tatsächlichen, am Outcome gemessenen Qualitätsverbesserung erzielt werden soll, hierauf sogar angewiesen. Anreizsysteme wie $\mathrm{P} 4 \mathrm{P}$ sollten deshalb nicht überstrapaziert oder als Alternativen anstelle „korporatistischer" Qualitätssicherungsmaßnahmen gehandelt, sondern ergänzend und dosiert zur Erreichung umschriebener Ziele eingesetzt werden zum Beispiel zur überfälligen Lösung von Schnittstellenproblemen an den Sektorengrenzen bzw. zur Förderung transsektoraler und berufsgruppenübergreifender Zusammenarbeit.

Autorenerklärung: Die Autorin erklärt, dass keine relevanten finanziellen Verbindungen in Bezug auf dieses Manuskript bestehen.

\section{Literatur}

1 Mannion $R$, Davies $H$. Payment for performance in health care. BMJ 2008; 336: 306-308

2 Norling $R$. Pay for Performance. Lessons for Healthcare leaders. International Quality Improvement Exchange Conference Haarlem, 2006

3 Sachverständigenrat zur Begutachtung der Entwicklung im Gesundheitswesen. Gutachten 2007: Kooperation und Verantwortung - Voraussetzungen einer zielorientierten Gesundheitsversorgung. http:// www.svr-gesundheit.de 